\title{
Camera-based objective measures of Parkinson's disease gait features
}

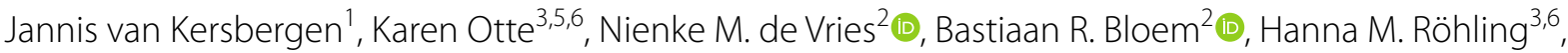 \\ Sebastian Mansow-Model ${ }^{3}$, Nicolien M. van der Kolk ${ }^{2} \mathbb{D}$, Sebastiaan Overeem ${ }^{1,4} \mathbb{C}$, Svitlana Zinger ${ }^{1}$ and \\ Merel M. van Gilst ${ }^{1,4^{*}}$ (1)
}

\begin{abstract}
Objective: Parkinson's disease is a common, age-related, neurodegenerative disease, affecting gait and other motor functions. Technological developments in consumer imaging are starting to provide high-quality, affordable tools for home-based diagnosis and monitoring. This pilot study aims to investigate whether a consumer depth camera can capture changes in gait features of Parkinson's patients. The dataset consisted of 19 patients (tested in both a practically defined OFF phase and ON phase) and 8 controls, who performed the "Timed-Up-and-Go" test multiple times while being recorded with the Microsoft Kinect V2 sensor. Camera-derived features were step length, average walking speed and mediolateral sway. Motor signs were assessed clinically using the Movement Disorder Society Unified Parkinson's Disease Rating Scale.

Results: We found significant group differences between patients and controls for step length and average walking speed, showing the ability to detect Parkinson's features. However, there were no differences between the ON and OFF medication state, so further developments are needed to allow for detection of small intra-individual changes in symptom severity.
\end{abstract}

Keywords: Parkinson's disease, Movement disorders, Kinect TUG-test

\section{Introduction}

Parkinson's disease (PD) is the second most common age-related neurodegenerative disease, which markedly affects patients' motor as well as a variety of non-motor functions. Various gait features are indicators of PD severity, such as a reduced step length, walking speed [1$4]$ and movement in the transverse plane $[5,6]$. The disease severity can be scored clinically using standardized assessments, such as the Movement Disorder Society Unified Parkinson's Disease Rating Scale (MDS-UPDRS) [7]. Administering this scale is time consuming and resource intensive for both patients and clinicians and is

\footnotetext{
${ }^{*}$ Correspondence: M.M.v.Gilst@tue.nl

1 Eindhoven University of Technology, 5612 AJ Eindhoven, The Netherlands

Full list of author information is available at the end of the article
}

also susceptible to intra- and inter-rater variability due to the subjective nature of clinical scoring [8].

Gait features can be measured objectively with 3D camera recordings. One promising low-cost consumer device is the Microsoft Kinect for Xbox One sensor (V2), which records RGB-depth data and tracks 25 anatomical landmarks in 3D space without the need for body-attached sensors. For an example, see Fig. 1A. Its accuracy and consistency have been validated with a gold standard system of optical motion capture (Vicon) $[9,10]$. In recent studies, Kinect derived kinematic features were used to detect group-level differences of motor patterns between healthy controls and persons with PD for fine motor tasks such as finger tapping [11] and gait tasks [12,13], including the "Timed Up-and-Go" (TUG) test [14]. However, these studies did not look at symptom severity changes within individuals with PD. 


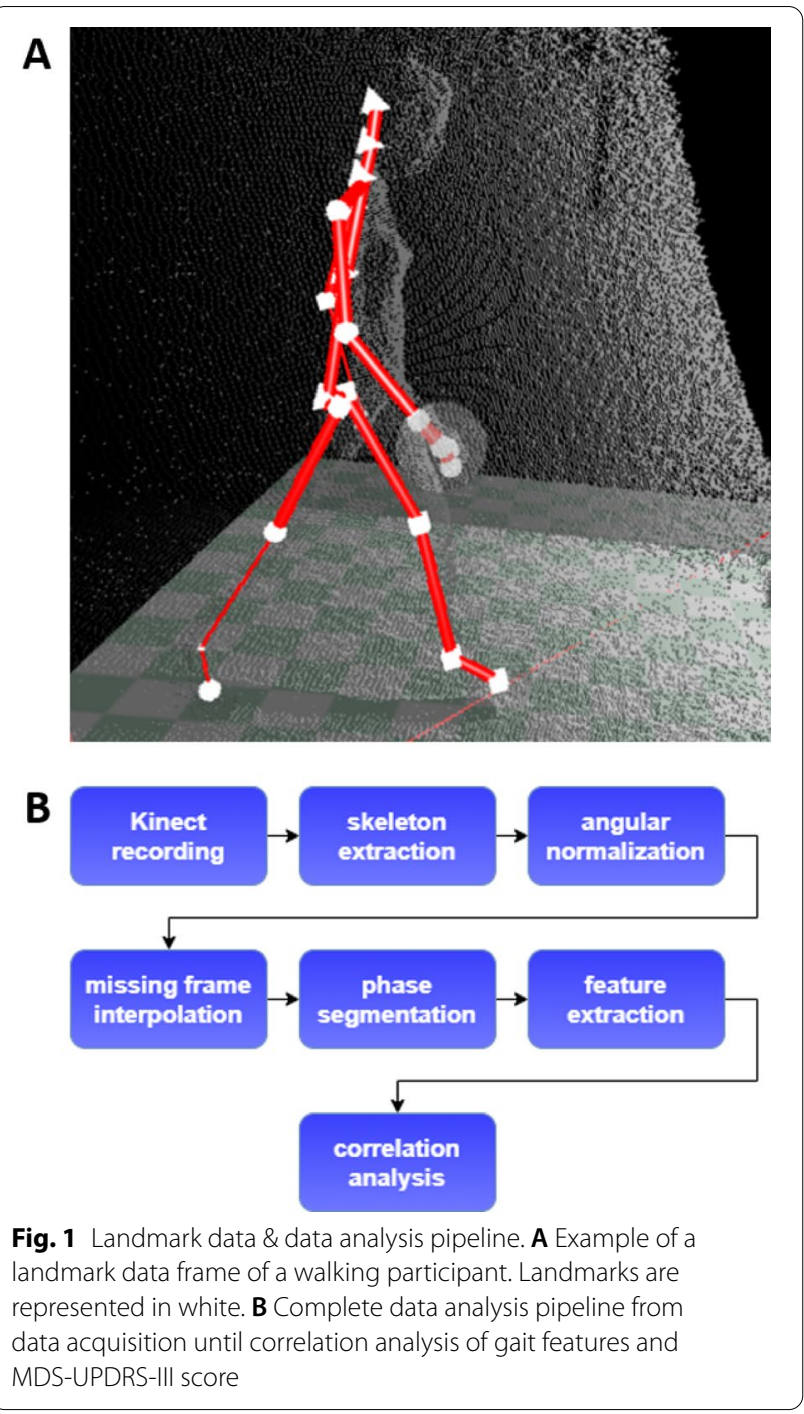

Detection of variation in individual symptom severity at home, could be a useful aid in monitoring disease progression and treatment effectiveness, giving clinicians time to focus on other aspects of patient care. Especially in times of a pandemic, benefits of home-based assessments become increasingly evident [15]. This would be especially valuable for persons with PD since considerable symptom fluctuations can be present, depending on factors such as the efficacy of medication, stress, fatigue or anxiety [16]. This study aims to investigate to what extent kinematic gait parameters, as derived with the Kinect, reflect intraindividual motor function differences $\mathrm{ON}$ and $\mathrm{OFF}$ medication, while performing a standard sequential motor task (TUG-test).

\section{Main text \\ Methods \\ Participants}

Data was collected in participants of the Park in Shape study [17], a randomized controlled trial evaluating the effectiveness of aerobic exercise on PD symptoms. The subjects mean age was $59.17 \pm 8.22$ years $(\mathrm{SD})$ ranging 30 to 75 years. Subjects had idiopathic PD diagnosed by a neurologist, with Hoehn and Yahr (H\&Y) stages I-II (tested off medication), no cognitive impairment (MiniMental State Examination > 24) and were stable on dopaminergic medication dose for at least 1 month. Exclusion criteria and a detailed design of the Park in Shape study can be found in the published protocol [17].

Healthy controls were recruited at the Radboud University Medical Center. The mean age was $34.57 \pm 12.18$ years (SD) with a range of 21 to 57 years. Exclusion criteria were neurodegenerative diseases or any other disease affecting motor function.

\section{Data collection}

Study procedure Participants abstained from taking their regular PD medication for $12 \mathrm{~h}$ before the first assessment (i.e. a practically defined OFF medication state). The motor symptoms were assessed using the MDSUPDRS-III and participants performed the TUG-test three times. Then, participants took their regular medication and repeated both the MDS-UPDRS-III and the TUG-test after $90 \mathrm{~min}$ (ON medication state).

Timed up-and-go test The TUG-test is a standard movement task to assess balance and mobility [18]. The task consists of five sequential motor sub-tasks: standing up from a seated position, standing up, walking three meters forward as fast as possible, turning around and walking back to sit down again.

Data acquisition Test performance was recorded with the Kinect V2 sensor (Microsoft, Redmond WA, USA), which is able to record RGB-depth data and track 25 joint positions of persons in frame with a framerate of $30 \mathrm{~Hz}$ within a range of $0.5 \mathrm{~m}$ up to $4.5 \mathrm{~m}$ with a depth resolution of $512 \times 424$ pixels and a field of view of $70.6^{\circ} \times 60^{\circ}$. The Kinect Sensor was placed shortly after the turning point of the TUG-test at roughly the participant's eye-level.

\section{Data analysis}

A flowchart of the data analysis pipeline is displayed in Fig. 1B. For this pilot study only the first walking phase of the TUG-test was used, since its signal to noise ratio was the highest. Both the stand-up and sit-down phase were at the edge of the Kinect sensor range and therefore more noise corrupted. The turning phase occurred at the other edge of the sensor range, where 
the landmark tracking especially for the legs appeared to be unreliable. The second walking phase was also excluded, since the Kinect landmark tracking assumes that a person faces the camera, leading to less precise estimations.

Data processing From each recording, we extracted the 3D landmark time-series using a software tool built and provided by Motognosis based on the Kinect software development kit [19].

To avoid confounding from differing camera positions, we normalized landmark data with respect to camera roll and pitch.

Landmark signals from missing frames were interpolated linearly.

In order to cleanly extract the first walking phase, we segmented recordings according to the following procedure. The walking phase is characterized by periodic increase and decrease of the anterior-posterior distance between the feet. Therefore, we defined the start of the walking phase as the frame, in which the distance between the ankles exceeded a threshold of 0.1 meters for the first time.

The walking phase ends, as the participants starts to turn around. Therefore we defined it as the frame in which the horizontal projection of the shoulder distance starts to decline strongly. This decline is best seen as a negative peak in the second derivative (acceleration) of the shoulder distance.

\section{Features}

We used three gait features: walking speed, step length and mediolateral sway. Features were extracted using the Motognosis Labs software (Motognosis $\mathrm{GmbH}$, Berlin), which was validated previously $[9,19]$ and averaged over all recordings of an individual per medication state. Additionally feature validation was done by visual inspection and outlier detection.

Walking speed The average walking speed was calculated by computing the time of participants walking phase and computing the Euclidean distance of the base of the spine from the first to the last frame.

Step length The step length is defined as the distance between the ankle landmarks, while both feet are on the ground. It was extracted with the Motognosis Labs software by finding the stance phases for both feet. To increase stance phase accuracy a velocity threshold was introduced and additional step length thresholds were implemented to filter out outliers.

Mediolateral sway We extracted the mediolateral sway by calculating the standard deviation of the mediolateral movement of the base of the spine.

\section{Statistical analysis}

To investigate the correlation between the MDS-UPDRSIII score and the gait features, we calculated Spearman's rank correlation coefficient for both the $\mathrm{ON}$ and $\mathrm{OFF}$ condition. Furthermore, we used t-tests (paired tests in case of patients in ON and OFF condition) to investigate the group differences per extracted feature. We calculated the effect size of group differences as Cohen's $d$. The statistical tests were not corrected for multiple comparisons as the study was explorative and descriptive in nature. Additionally the group difference in MDS-UPDRS-III score was calculated to ensure that disease severity differed significantly between the ON and OFF state.

\section{Results}

Data

We included 19 persons with PD and 8 healthy controls.

Of the 145 total recordings, 128 recordings were used for analysis. We excluded recordings if the landmark data could not be extracted, the participant ran during the walking phase or if the participant had an atypical response to the medication, where the MDS-UPDRS-III score increased after taking medication. More recordings from TUG tests performed in ON state than in OFF state had to be excluded leading to the following data distribution:

- PD OFF: 60 recordings from 18 persons

- PD ON: 44 recordings from 12 persons

- control 24 recordings from 8 persons

\section{Main results}

The main findings are displayed in Table 1. The MDSUPDRS-III group difference between ON and OFF group was significant (ON: $24.5 \pm 14.4$ vs. OFF: $34.9 \pm 15.5$, $\mathrm{p}<0.0001$ ), indicating a clinical discernible medication effect. There were significant group differences between PD patients (both ON and OFF) and controls, for the features step length and walking speed with effect sizes larger than 1 for all comparisons. Within the PD group, intra-individual differences and gait parameters between the ON and OFF state were not statistically significant.

For associations between extracted features and disease severity, only the correlation of the mediolateral sway with the MDS-UPDRS-III score in the OFF group was significant $(\mathrm{r}=0.599, \mathrm{p}=0.01)$.

Fig. 2 shows the MDS-UPDRS-III score plotted against the step length for participants in ON and OFF state. For six of the twelve participants (with available ON and OFF recordings), step length increased after taking medication. 
Table 1 Results

\begin{tabular}{lccc}
\hline Metric & Step length $(\mathbf{m})$ & Avg. speed (m/s) & Mediolateral sway (cm) \\
\hline Mean HC (SD) & $0.776(0.091)$ & $1.357(0.218)$ & $1.544(0.690)$ \\
Mean OFF (SD) & $0.613(0.102)$ & $1.024(0.192)$ & $1.692(0.526)$ \\
Mean ON (SD) & $0.664(0.118)$ & $1.005(0.186)$ & $1.787(0.610)$ \\
Difference OFF-HC (rel. diff.) & $-0.16(5.84 \%)$ & $-0.333(6.98 \%)$ & $0.148(2.29 \%)$ \\
Difference ON-HC (rel. diff.) & $-0.111(3.87 \%)$ & $-0.351(7.44 \%)$ & $0.243(3.65 \%)$ \\
Difference OFF-ON (rel. diff.) & $-0.051(2.00 \%)$ & $0.019(0.46 \%)$ & $-0.095(1.36 \%)$ \\
p-value of t-test OFF-HC (Cohen's d) & $0.001(-1.643)$ & $<0.001(-1.665)$ & $0.552(0.256)$ \\
p-value of t-test ON-HC (Cohen's d) & $0.047(-1.004)$ & $0.001(-1.797)$ & $0.418(0.383)$ \\
p-value of t-test OFF-ON (Cohen's d) & $0.218(-0.461)$ & $0.792(0.010)$ & $0.654(-0.166)$ \\
Corr. coef. OFF with UPDRS (p-value) & $0.08(0.77)$ & $0.01(0.97)$ & $0.599(0.01)$ \\
Corr. coef. ON with UPDRS (p-value) & $-0.530(0.09)$ & $-0.260(0.44)$ & $0.361(0.28)$ \\
\hline
\end{tabular}

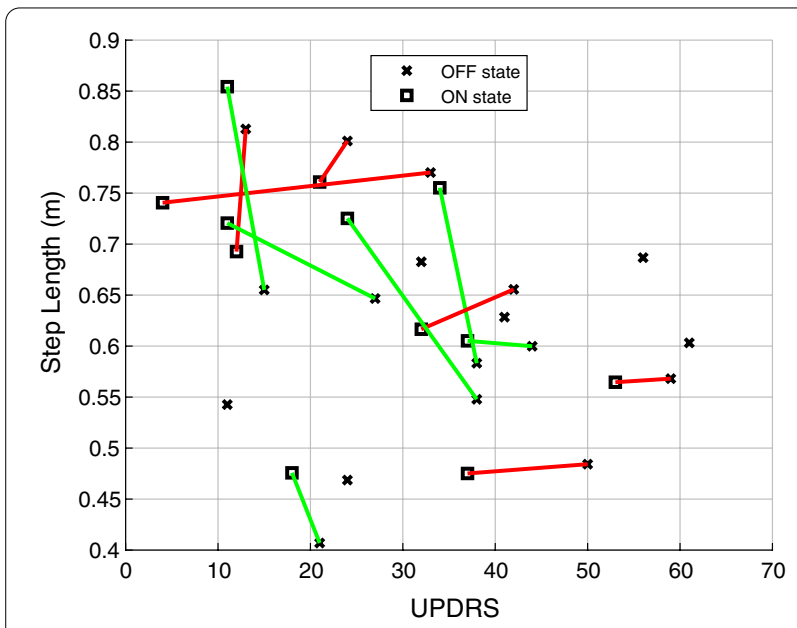

Fig. 2 Comparison of step length parameter in ON and OFF state, lines connect measurements in ON and OFF state. Red lines represent a decreased step length after taking medication, green lines represent an increased step length

\section{Discussion}

In our sample, the Kinect Sensor in combination with Motognosis Labs software was capable of extracting several gait features in PD patients during a functional mobility test. There were significant differences in step length and average speed between patients with PD and controls, with a large effect size (Cohen's $d>1$ ). This confirms that the Kinect sensor is a promising tool to quantify PD-specific gait behaviour [2, 4, 6]. However, although a medication effect could be detected clinically, there were no significant differences in task features between the OFF and ON conditions.

This suggests that the current setup is not sufficiently sensitive to relatively small differences in motor functioning between the $\mathrm{ON}$ and OFF condition in persons with mild symptoms (only H\&Y stages I and II included). However, in almost all participants the step length either increased or did not change strongly in the ON condition, indicating a trend towards an increased step length for reduced MDS-UPDRS-III scores, which is in line with previous research $[1,2$, 14]. Interestingly, the strongest correlation with MDSUPDRS-III was found for mediolateral sway. This was not expected, since Parkinsonian gait is characterized by stiffness and decreased transversal motion. A possible explanation might be the compromised balance of people with PD $[20,21]$ resulting in more compensatory movements.

The current study may well have been underpowered to detect differences intraindividual gait differences in $\mathrm{ON}$ versus OFF. We should also point out that we included patients with relatively mild disease, where the differences between the ON and the OFF state, although clinically evident, were not very large. Possibly some of these differences were caused by symptoms that are not directly reflected in gait. Much larger differences are typically seen in more advanced patients who experience response fluctuations, and it is possible that quantitative gait analysis using the remote kinematic analysis can detect relevant differences in such a more severely affected population. However, the practical use of these camera systems would mainly lie in ambulatory follow-up of disease progression or medication effect, necessitating sufficient sensitivity on an individual level. Since PD is a complex disease with a very heterogeneous presentation, increasing the number of outcome parameters and combining them in a single model may enable better detection of intra-individual differences. Using an adapted version of the TUG, such that the whole sequence can be successfully parametrized, or additional instrumentally assessed, standardized motor tasks such as the Short Physical 
Performance Battery [22] could expand on the kinematic features. Because the hypothesized intra-individual differences are likely small, standardized movement tests have an advantage over free movement by producing comparable, clean data and forcing a range of different movement patterns.

\section{Conclusion}

We were able to detect group differences in gait features between people with PD and healthy controls using the Kinect depth camera. However, the current task setup and analysis approach lacks sensitivity to detect small intra-individual changes in symptom severity.

\section{Limitations}

Limitations of this study include the small sample size, subjects with relatively mild symptoms and a not complete age match with the control population. The standard outcome for the TUG (task duration) could not be analysed because of missing frames at the beginning of the recording.

\section{Abbreviations}

PD: Parkinson's disease; TUG: Timed Up-and-Go; MDS-UPDRS: Movement Disorders Society Unified Parkinson's Disease Rating Scale.

\section{Acknowledgements}

Not applicable.

\section{Authors' contributions}

JVK performed the analysis, was a major contributor in interpreting the results and was the main contributor in writing the manuscript. KO, HMR and SMM wrote and made available the Motognosis Labs algorithm and aided in the interpretation of the results. NMV, BRB, MMG and SO designed the study. NMV and NK collected the data. SZ, SO and MMG were major contributors in interpreting the results and writing the manuscript. All authors reviewed the manuscript. All authors read and approved the final manuscript.

\section{Funding}

This study was supported by a VIDI research grant from the Netherlands Organization for Scientific Research (Grant No. 016.116.371) to SO.

\section{Availability of data and materials}

The datasets generated and analysed during the current study are available from the authors upon reasonable request. There are limitations with regard to raw $R G B$ and depth data because of identifiability of patients.

\section{Declarations}

\section{Ethics approval and consent to participate}

The study protocol was approved by the medical ethics committee of Radboud University Medical Center, Nijmegen, the Netherlands. All subjects provided written informed consent.

\section{Consent for publication}

Not applicable.

\section{Competing interests}

$\mathrm{KO}, \mathrm{HMR}$ and SMM work at Motognosis GmbH. The company was not involved in the study design and did not have influence on the decision to publish. All other authors declare that they have no competing interests.

\section{Author details}

${ }^{1}$ Eindhoven University of Technology, 5612 AJ Eindhoven, The Netherlands. ${ }^{2}$ Department of Neurology, Radboud University Medical Center, Geert Grooteplein Zuid 10, 6525 GA Nijmegen, The Netherlands. ${ }^{3}$ Motognosis GmbH, Schönhauser Allee 177, 10119 Berlin, Germany. ${ }^{4}$ Sleep Medicine Center Kempenhaeghe, Sterkselseweg 65, 5591 VE Heeze, The Netherlands. ${ }^{5}$ NeuroCure Clinical Research Center, Charité - Universitätsmedizin Berlin, Corporate Member of Freie Universität Berlin, Humboldt-Universität zu Berlin, and Berlin Institute of Health, Berlin, Germany. ${ }^{6}$ Experimental and Clinical Research Center, Charité - Universitätsmedizin Berlin Corporate Member of Freie Universität Berlin, Humboldt-Universität zu Berlin, and Berlin Institute of Health and Max Delbrück Center for Molecular Medicine, Berlin, Germany.

Received: 29 March 2021 Accepted: 16 August 2021

Published online: 26 August 2021

\section{References}

1. Nelson A, Zwick D, Brody S, Doran C, Pulver L, Rooz G, Sadownick M, Nelson R, Rothman J. The validity of the GaitRite and the functional ambulation performance scoring system in the analysis of Parkinson gait. NeuroRehabilitation. 2002;17:255-62.

2. Williams A, Peterson D, Earhart G. Gait coordination in Parkinson disease: effects of step length and cadence manipulations. Gait Posture. 2013:38:340-4.

3. Kimmeskamp S, Hennig E. Heel to toe motion characteristics in Parkinson patients during free walking. Clin Biomech. 2001;16:806-12.

4. Bohnen N, Frey K, Studenski S, Kotagal V, Koeppe R, Scott P, Albin R, Müller M. Gait speed in Parkinson disease correlates with cholinergic degeneration. Neurology. 2013;81:1611-6.

5. Murray M, Sepic S, Gardner G, Downs W. Walking patterns of men with parkinsonism. Am J Phys Med. 1987;57:278-94.

6. Morrs M, Huxham F, McGinley J, Dodd K, lansek R. The biomechanics and motor control of gait in Parkinson disease. Clin Biomech. 2001;16:459-70.

7. Movement Disorder Society Task Force on Rating Scales for Parkinson's Disease. The unified Parkinson's disease rating scale (UPDRS): status and recommendations. Mov Disord. 2003;81:738-50.

8. Post B, Merkus MP, de Bie RM, de Haan RJ, Speelman JD. Unified Parkinson's disease rating scale motor examination: are ratings of nurses, residents in neurology, and movement disorders specialists interchangeable? Mov Disord. 2005;20:1577-84.

9. Otte K, Kayser B, Mansow-Model S, Verrel J, Paul F, Brandt A, SchmitzHübsch T. Accuracy and reliability of the kinect version 2 for clinical measurement of motor function. PLoS ONE. 2016;11:e0166532.

10. Clark RA, Pua YH, Oliveira CC, Bower KJ, Thilarajah S, McGaw R, Hasanki K, Mentiplay BF. Reliability and concurrent validity of the Microsoft Xbox One Kinect for assessment of standing balance and postural control. Gait Posture. 2015;42:210-3.

11. Buongiorno D, Bortone I, Cascarano GD, Trotta GF, Brunetti A, Bevilacqua $\checkmark$. A low-cost vision system based on the analysis of motor features for recognition and severity rating of Parkinson's disease. BMC Med Inform Decis Making. 2019;19:243.

12. Eltoukhy M, Kuenze C, Oh J, Jacopetti M, Wooten S, Signorile J. Microsoft kinect can distinguish differences in over-ground gait between older persons with and without Parkinson's disease. Med Eng Phys. 2017;44:1-7.

13. Dranca L, de Mendarozketa LDAR, Goni A, Illarramendi A, Gomez IN, Alvarado MD, Rodiguez-Oroz MC. Using kinect to classify Parkinson's disease stages related to severity of gait impairment. BMC Bioinform. 2018;19:471.

14. Tan D, Pua Y-H, Balakrishnan S, Scully A, Bower KJ, Prakash KM, Tan E-K, Chew J-S, Poh E, Tan S-B, Clark RA. Automated analysis of gait and modified timed up and go using the Microsoft Kinect in people with Parkinson's disease: associations with physical outcome measures. Med Biol Eng Comput. 2019;57:369-77.

15. Bloem BR, Dorsey ER, Okun MS. The coronavirus disease 2019 crisis as catalyst for telemedicine for chronic neurological disorders. JAMA Neurol. 2020;77:927-8

16. Jankovic J. Motor fluctuations and dyskinesias in Parkinson's disease: clinical manifestations. Mov Disord. 2005;20:511-6.

17. Van der Kolk NM, Overeem S, De Vries NM, Kessels RP, Donders R, Brouwer $M$, Berg D, Post B, Bloem BR. Design of the park-in-shape study: a phase 
II double blind randomized controlled trial evaluating the effects of exercise on motor and non-motor symptoms in Parkinson's disease. BMC Neurol. 2015;15:56.

18. Podsiadle D, Richardson $S$. The timed up and go: a test of basic functional mobility for frail elderly persons. J Am Geriatr Soc. 1991;39:142-84.

19. Steinert A, Sattler I, Otte K, Röhling H, Mansow-Model S, Müller-Werdan U. Using new camera-based technologies for gait analysis in older adults in comparison to the established GaitRite system. Sensors. 2020;20:125.

20. Ebersbach G, Edler D, Kaufhold O, Wissel J. Whole body vibration versus conventional physiotherapy to improve balance and gait in Parkinson's disease. Phys Med Rehabilitat. 2008;89:399-403.

21. Gobbi L, Oliveira-Ferreira M, Caetano M, Lirani-Silva E, Barbieri F, Stella F, Gobbi S. Exercise programs improve mobility and balance in people with Parkinson's disease. Parkinsonism Relat Disord. 2009;1553:49-52.
22. Guralnik JM, Simonsick EM, Ferrucci L, Glynn RJ, Berkman LF, Blazer DG, Scherr PA, Wallace RB. A short physical performance battery assessing lower extremity function: association with self-reported disability and prediction of mortality and nursing home admission. J Gerontol. 1994:49:85-94.

\section{Publisher's Note}

Springer Nature remains neutral with regard to jurisdictional claims in published maps and institutional affiliations.
Ready to submit your research? Choose BMC and benefit from:

- fast, convenient online submission

- thorough peer review by experienced researchers in your field

- rapid publication on acceptance

- support for research data, including large and complex data types

- gold Open Access which fosters wider collaboration and increased citations

- maximum visibility for your research: over $100 \mathrm{M}$ website views per year

At BMC, research is always in progress.

Learn more biomedcentral.com/submissions 